\title{
Pattern-based Automatic Translation of Structured Power System Data to Functional Models for Decision Support Applications
}

\author{
Heussen, Kai; Weckesser, Johannes Tilman Gabriel; Kullmann, Daniel
}

Published in:

Proceedings of IWIES 2013

Link to article, DOI:

10.1109/IWIES.2013.6698579

Publication date:

2013

Link back to DTU Orbit

Citation (APA):

Heussen, K., Weckesser, J. T. G., \& Kullmann, D. (2013). Pattern-based Automatic Translation of Structured Power System Data to Functional Models for Decision Support Applications. In Proceedings of IWIES 2013 IEEE. https://doi.org/10.1109/IWIES.2013.6698579

\section{General rights}

Copyright and moral rights for the publications made accessible in the public portal are retained by the authors and/or other copyright owners and it is a condition of accessing publications that users recognise and abide by the legal requirements associated with these rights.

- Users may download and print one copy of any publication from the public portal for the purpose of private study or research.

- You may not further distribute the material or use it for any profit-making activity or commercial gain

- You may freely distribute the URL identifying the publication in the public portal 


\title{
Pattern-based Automatic Translation of Structured Power System Data to Functional Models for Decision Support Applications
}

\author{
Kai Heussen, Johannes T. G. Weckesser, Daniel Kullmann \\ Department of Electrical Engineering \\ Technical University of Denmark \\ Email: \{kh, jtgw, daku\}@elektro.dtu.dk
}

\begin{abstract}
Improved information and insight for decision support in operations and design are central promises of a smart grid. Well-structured information about the composition of power systems is increasingly becoming available in the domain, e.g. due to standard information models (e.g. CIM or IEC61850) or otherwise structured databases. More measurements and data do not automatically improve decisions, but there is an opportunity to capitalize on this information for decision support. With suitable reasoning strategies data can be contextualized and decision-relevant events can be promoted and identified. This paper presents an approach to link available structured power system data directly to a functional representation suitable for diagnostic reasoning. The translation method is applied to test cases also illustrating decision support.
\end{abstract}

Keywords-power system modeling, knowledge-based methods, functional modeling, model translation, decision support

\section{INTRODUCTION}

Operational complexity of power systems is increasing, partly due to the increasing penetration of fluctuating renewable energy sources (RES) and the active utilization of the demand side flexibility. At the same time the trend toward smarter grids is generating more and diverse data, which in turn increases the information complexity to be dealt with. Decision making becomes harder. On the other hand, the increasing focus on data, information and interoperability has lead to an increased availability of well-structured information models and correspondingly more well-organized data retrieval. For example the CIM-related and IEC61850 standards provide consistent categories and principles for modeling data in a structured fashion [1], [2]. While the IEC61850 data structures provide an object-oriented modeling logic, the CIM family additionally provides formalized relationships between attributes and thus can be interpreted as ontology [3]. Other automation standards being introduced in the related automation disciplines such as the OPC-UA or IEC61499 [4], [5] also are supported by or easily enhanced with semantic technologies. Along with the introduction of such standards and other ontology-based modeling concepts, methods for the handling, consistency checking and transformation of such data have been introduced [6].

These data structures allow to retain the information value of data generated, stored and retrieved in disparate physical and virtual locations by providing a generic standardized context. The generic context given by the information model often is not the context required for a specific system function, where data from different sources usually needs to be combined. This function-oriented data context can also be modelled in an ontology, which has bee termed "functional ontology" in [7]. The translation from source- to application data can then be expressed as ontology mapping, and automated using semantic technologies.

Structured conceptual and information modelling has a tradition in context of system engineering, due to often complex data interdependencies and strong focus on information consistency. Here, functional modeling focuses on the description and relations of functions performed by components, subsystems or entire systems which relates to both requirements engineering (e.g. [8]) and decision support domains (e.g. [9]). Operations on data aggregation, simplification, expansion and performance specifications are supported by a functional modeling perspective [10], [11]. As opposed to function blocks, which define semantics for controls specification (IEC61499, discussed in [5]), functional models identify functional relations and support reasoning about system functions.

The functional modeling language employed in this work is Multilevel Flow Modeling (MFM) which has been developed with a focus on decision support. MFM defines a well developed set of domain-independent functions and abstractions which can be applied to several technology domains and application categories [12]. Offering strong first principles for the models, it is well-suited for e.g. diagnostic reasoning [13] or other decision support functions. As MFM provides domain-independent modeling concepts, reasoning algorithms and software can be developed independently from specific applications. A conventional MFM application is thus based on (i) model development, (ii) data engineering and (iii) application testing and visualization.

MFM is well suited for model-translation due to its strong formal properties and domain-independence of its core concepts. This paper presents the first realized application of pattern mapping and model translation using MFM, thereby collapsing the manual application engineering steps (i) and (ii) into one automated step. This automation enables both more rapid deployment and scaling to larger applications beyond the reach of a manual modeling process. Section II introduces the overall approach; Section III provides an illustration of the pattern developed and mapping and Section IV outlines the model translation algorithm. In Section V, the application of 


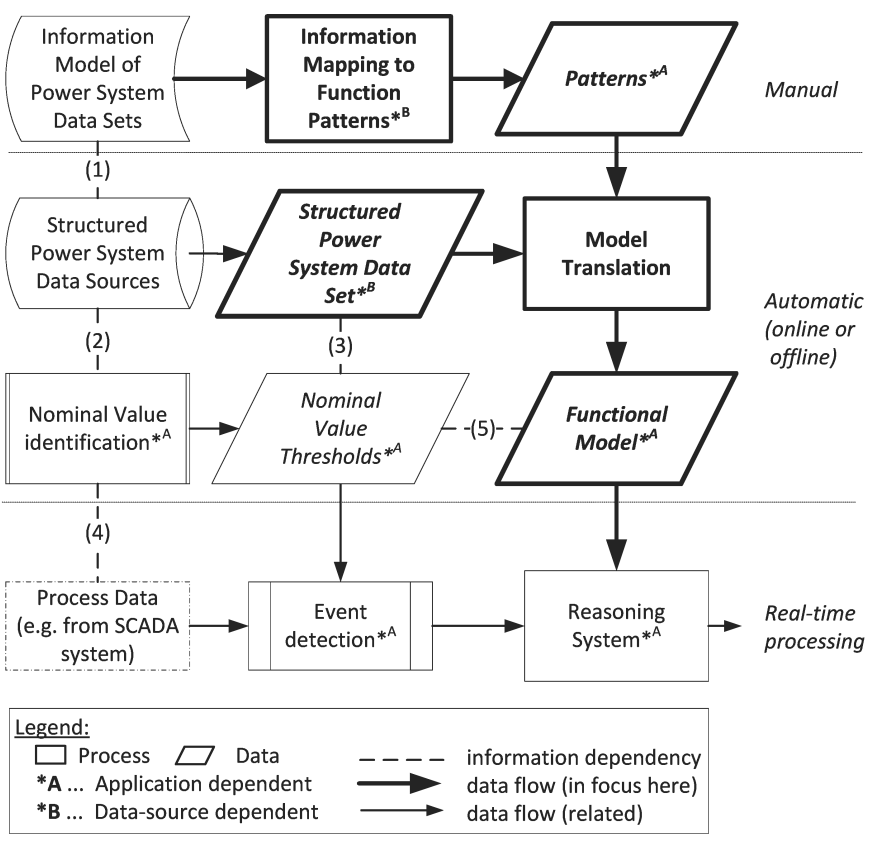

Fig. 1. Data flow chart of the model translation from power system data set to a functional model, presented in context of an event-based reasoning application. The numbered dashed lines indicate the following relations: (1)instance-of; (2),(3),(5) - refers to; (4)- representation of.

the generated MFM models for reasoning is illustrated.

\section{BACKGROUND AND APPROACH}

This section provides an overview of the model translation and reasoning approach suggested in this paper and relates this approach to an application context. Potential data sources are listed and characterized. Finally, a short introduction to the functional modeling approach employed here, Multilevel Flow Modeling (MFM), is provided.

\section{A. From Data Model to Reasoning Model}

The overall concept and context of the model translation approach presented in this work is illustrated in Figure 1. The bold outlines emphasize the core aspects addressed in this paper. The chart indicates process steps and types of data as well as two kinds of relations: a data processing flow, indicated by arrows, and informational dependencies, indicated by dashed lines. The processing steps are organized from upper left to lower right. The information dependencies indicate different kinds of logical dependencies of the information content. The existence of these relations is fundamental for the translation process.

Vertically, the chart is three-layered to characterize the type of processing involved. In the upper layer, the pattern identification and mapping to a specific (standardized) information model is considered a manual process of knowledge engineering, further illustrated in Section III. Selection and translation of a specific power system data set and model translation are automatic processes which are introduced in Section IV. Finally the functional model is employed for fast abductive reasoning e.g. to infer diagnostic knowledge from actual process data.
To consider the engineering effort in a specific application, it is indicated which process steps are application dependent $\left({ }^{*} \mathrm{~A}\right)$ and which are dependent upon the data source $\left({ }^{*} \mathrm{~B}\right)$. The functional ontology, here MFM, is employed both in the information mapping to function patterns and the actual model translation process. MFM can be applied in many engineering domains and the relevant MFM patterns to be generated depend both on the domain and on the objective of the reasoning application (see also Section II-C and V).

\section{B. Data Sources}

The information and data sources sources for model translation have two main parts: the ontology or fixed data model of the power system data set and the actual case specific data. In case of CIM [14] and IEC61850 [1] based data sets, structured data models are provided by the respective standard. The existence of an ontology is not essential here, but the modeling principle employed in defining the respective structured data set. If data sets that do not have a standard-based ontology are considered, it is still possible that similar basic modeling principles have been applied.

In this paper, the data source in consideration follows the a common modeling principle in power systems using buses and lines, which is also associated with one-line diagrams. The data structure of the source data is based on the IEEE Common Data Format for the Exchange of Solved Load Flow Data [15]. The same modeling principles, however, can also be identified e.g. in the CIM wires package.

\section{Functional Modeling and MFM}

Functional modeling - or function modeling - is a form of conceptual modeling aiming to represent the (design- or use-) functions of a system. The function of an object is the attribution of a purpose in context of some action, and as such, a function thus describes a directed mapping between an object and a purpose. Explicit representation of functions is used e.g. in requirements engineering to formulate a system composition, interactions and to associate performance requirements with system functions. Another use of functional models is in reasoning about causes and consequences, e.g. for failure mode and effect analysis or diagnostic reasoning for operator support.

Multilevel Flow Modeling (MFM) [16] is an approach to modeling goals and functions of complex industrial processes involving interactions between controls and flows of mass or energy. The MFM concepts, presented in Figure 2, can be composed to develop a model of purpose \& function of complex processes, capturing several levels of abstraction. A tutorial introduction to MFM can be found in [17], for control functions in [18] and to related cause/consequence reasoning in [13]. MFM is supported by tools for model building and reasoning: a graphical modeling environment and a rule-based reasoning environment (MFM Workbench). An overview of the MFM tool-chain and review applications with references is found in [12]. Apart from the graphical notation, several data formats have been specified to exchange textual representations of an MFM model (see also Section IV-B). Application of MFM to Power Systems has been introduced in [19] with focus on model concepts and has been extended to a comprehensive set of model patterns in [10]. 


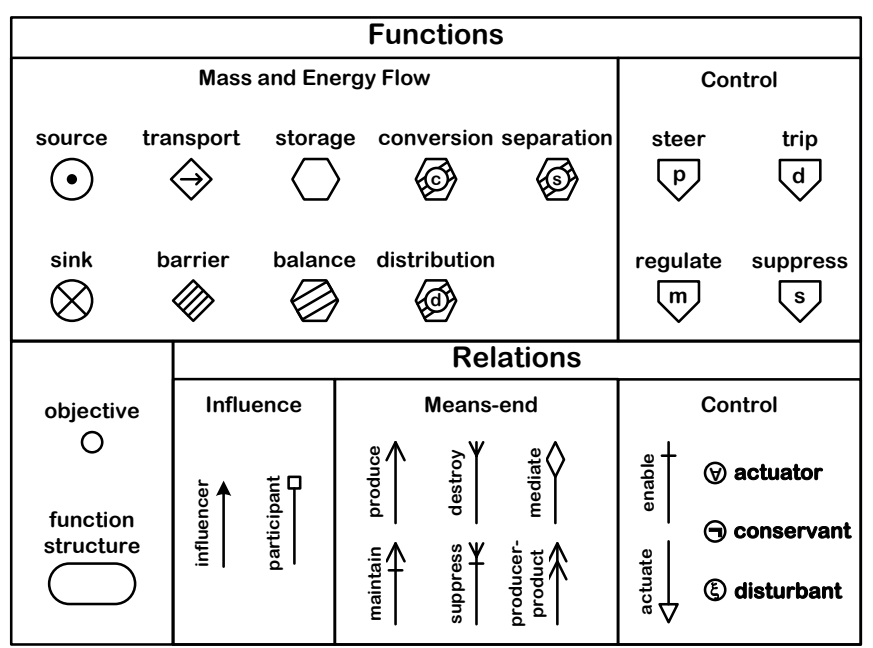

Fig. 2. MFM Concepts.

\section{MAPPING OF POWER SySTEM INFORMATION tO MFM PATTERNS}

In the following the mapping of power system information into MFM patterns is illustrated. In an information sciences context, this process can be considered as 'ontology mapping' [7], however, in the present work the focus is on modeling relations and information science aspects are secondary.

\section{A. Model Development on Example}

As the patterns employed for the model-translation are domain- and application specific, we start the illustration by identifying a set of patterns from an example with a specific modeling objective: to represent a power system active power flow in a steady state condition to support root cause reasoning about state deviations. A representation of the dynamics of the individual components is not intended. Further patterns e.g. to account for some of the dynamic characteristics or to consider also bi-directional flows are found in [10].

Figure 3 shows a one-line diagram of a simple three bus system with three branches. It contains two generators and two loads. Additionally, the solved power flow data are provided, consisting of the complex bus voltages, line flows and slack bus generation. The MFM model, shown in Figure 4 , was developed to capture causal relations with respect to active power flow and with the steady state flow employed as nominal flow. While for the power flow calculation losses were considered, the losses can be neglected in the MFM model due to their minor contributions. The complete MFM model includes an energy flow structure efs 1 containing the functions, including the association of objectives with each of the transport functions, representing a desired system state. Control functions are not employed. This MFM model already suggests some direct relations between the concepts in the oneline diagram and MFM function groups. In the following, these relations are isolated as individual patterns.

\section{B. MFM patterns for Power System Concepts}

In order to automatically generate such an MFM model from a power system data set, patterns representing the individual components need to be identified.

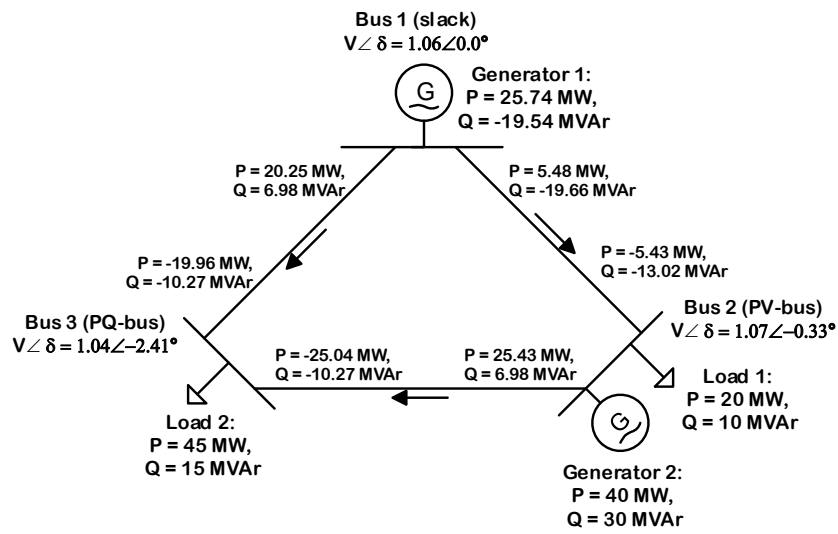

Fig. 3. One-line diagram of three bus system with solved load flow data.

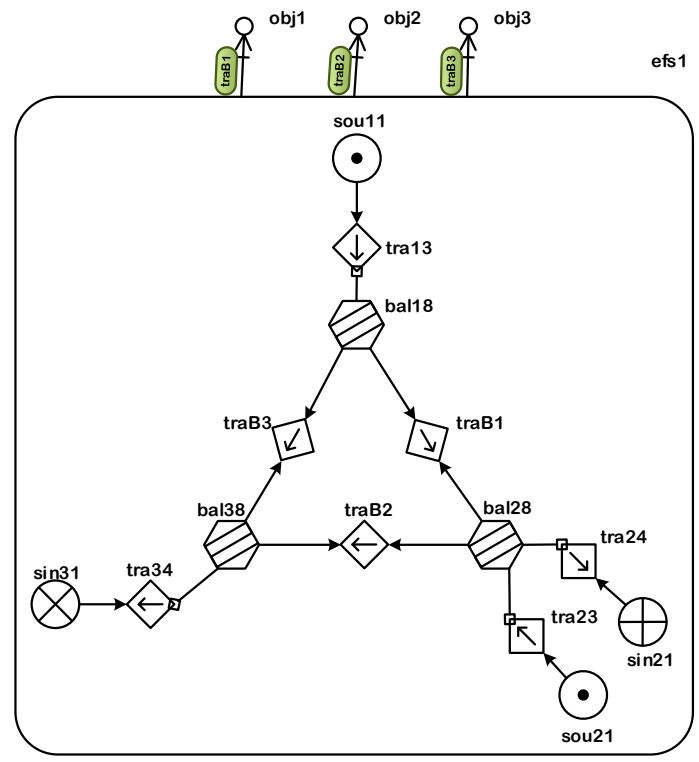

Fig. 4. Three bus system as MFM model.

1) Bus: In power systems the bus concept refers to a network point with a common voltage to which loads or generators are connected. These nodes are interconnected through transmission lines, cables or transformers. Here a bus is represented in MFM by a balance. According [17] a balance in MFM is a function that balances between the total rates of inflow and outflow. Figure 5 shows on the left hand-side the bus symbol and on the right the representation in MFM.

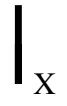

(a)

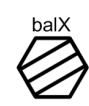

(b)
Fig. 5. Representation of a bus in 5a one line diagram and 5b MFM

2) Generation unit: A generation unit is a component that injects power into the grid. The pattern chosen to represent a power generation unit is shown in Figure 6). This pattern represents a synchronous generator driven by a steam turbine 
or the power injection at a point of common coupling (PCC) of any kind of electric generation facility, such as wind turbines or photovoltaic. The injection of power is represented by the

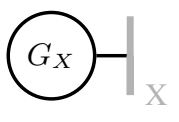

(a)

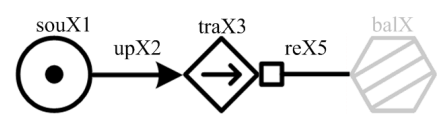

(b)
Fig. 6. Representation of a generator in $6 \mathrm{a}$ one line diagram and $6 \mathrm{~b}$ MFM

source function souX1. The connection and power transmission to the next bus is realized by the transport function traX3. The source and the transport are connected via an influencer and the balance representing the bus is connected with a participant (i.e. is passive w.r.t. transport flow state), so that the source state determines the flow through the transport (power injection) while the balance will not influence the transport flow.

3) Load: The load components are representing all power consuming devices. These can be as simple as an ohm resistive load (Figure 7b) or somewhat more complex like an electric machine load (Figure 7a). The corresponding MFM model consists of a sink and a transport function (Figure 7c). The

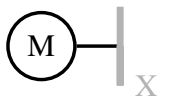

(a)

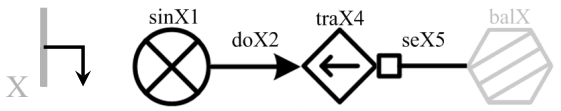

(b) (c)
Fig. 7. Representation of a classic load in $7 \mathrm{~b}$ one line diagram and 7c MFM

power consumption is represented by the sink function $\sin X 1$. The connection to the bus and the power transfer are realized by the transfer function. The causality of the energy flow is analogous to the Generation unit pattern.

4) Transmission line: In a lossless power system a transmission line's exclusive function is to transfer power/energy. Figure $8 \mathrm{~b}$ shows the mapping of a transmission line into MFM. The causal influence relations are of influencer type on both sides, since the effective energy flow is influenced by the surrounding system. As here the transfer function is thought of

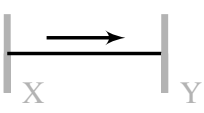

(a)

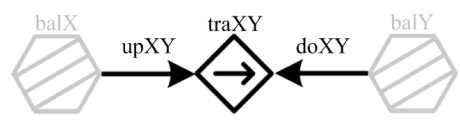

(b)
Fig. 8. Representation of a transmission line in $8 \mathrm{a}$ one line diagram and $8 \mathrm{~b}$ MFM

as associated with a nominal flow, the direction of the transfer function is chosen corresponding to numerical reference power flow direction during model building.

5) Functional Objectives: To complete the MFM model, the generated and interconnected function patterns need to be embedded in a flow-structure and objectives need to be associated with some main functions. As the present use case requires monitoring of the nominal flows in the transmission lines, an objective is associated with each transport function representing a transmission line. The corresponding pattern is shown in Figure 9.

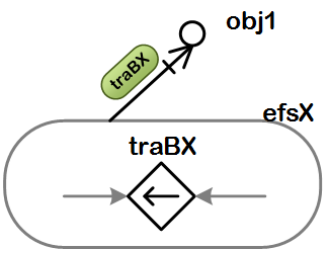

Fig. 9. Objective associated with lines for observing flow deviations.

\section{MOdel Translation}

In this section the method is illustrated on the IEEE Common Data Format (CDF) for the Exchange of Solved Load Flow Data [15] which thus allows to extract both topological information and numerical data for calculating the energy flow direction.

\section{A. Structured Power System Data}

The data provided in the CDF format is organized according to the principles of a one-line diagram as discussed in Section III, except that the bus data does not differentiate between different types of generators or loads. Consequently, all generators and loads are modeled as shown in Figures $6 \mathrm{a}$ and $7 \mathrm{~b}$, respectively. The IEEE format offers detailed information about transformers and phase shifters, which in this work are modelled as a transmission line.

The data provided in the CDF format for each bus and branch are presented in Table I and II, respectively. The bus

TABLE I. Bus DATA GIVEN In THE IEEE COMMON DATA Format

\begin{tabular}{|c|c|}
\hline Bus Data & Description \\
\hline Bus Number & four digit number used in the load flow \\
\hline Bus Name & $\begin{array}{c}\text { maximum } 12 \text { characters; suggested: } \\
\text { Area } 6-7, \text { name } 8-14 \text { and } \mathrm{kV} 15-17\end{array}$ \\
\hline Bus Area & $\begin{array}{c}\text { two digit integer, region where bus is located } \\
\text { 0 indicates unavailable data }\end{array}$ \\
\hline Bus Type & $\begin{array}{c}\text { Type 0: Unregulated bus, Load Bus (PQ-bus) } \\
\text { Type 1: reactive power generation within limits } \\
\text { Type 2: Regulated Generator Bus (PV-bus) } \\
\text { Type 3: Reference Bus (Swing Bus) }\end{array}$ \\
\hline Final Voltage & final bus voltage in $p . u$. \\
& from solved load flow calculation \\
\hline Angle & Final voltage angle in degrees \\
\hline Load & active and reactive power demand of load \\
\hline Generation & final active and reactive generation \\
\hline Capacitors & Ond Reactors \\
and & Optional: appropriate $k \mathrm{~V}$ base \\
\hline Loss Zone & \\
\hline Base &
\end{tabular}

data provide sufficient information to determine the number of buses and the locations where generators or loads are connected. Information on the network topology, the interconnection between the buses, is provided by the branch data. Furthermore, the power flow in each transmission can be determined utilizing the complex bus voltages given in the bus data and the complex line impedances given in the branch data, which is employed in the model translation to determine the nominal flow direction of the MFM transport function. 
TABLE II. BRANCH DATA GIVEN IN THE IEEE COMMON DATA FORMAT

\begin{tabular}{|c|c|}
\hline Branch Data & Description \\
\hline $\begin{array}{c}\text { Terminal } \\
\text { Identification }\end{array}$ & "from" and "to" bus numbers \\
\hline Circuit Number & numbering of parallel lines \\
\hline $\begin{array}{c}\text { Branch } \\
\text { Impedances }\end{array}$ & $R+j * X$ in $p . u$. \\
\hline Line Charging & Total line charging in $p . u$. \\
\hline Branch Type & $\begin{array}{c}\text { Type 0: transmission line } \\
\text { for more information see [15] }\end{array}$ \\
\hline Line Area & Optional: Region the branch is located \\
\hline Loss Zone & Optional: see [15] \\
\hline Line Ratings & $\begin{array}{c}\text { space for three apparent power ratings } \\
\text { with the first being the lowest }\end{array}$ \\
\hline
\end{tabular}

\section{B. Functional Model Specification}

For information processing purposes, the MFM graphical concepts presented in Figure 2 can be organized into an ontology or class concept representation which is employed for reasoning programs. For data exchange purposes, a simple graph-oriented (entity-relationship) notation is available which internally is translated to the complex class. The simpler notation is also generated here and consists of generic typed nodes and three relations: link, whole-part and attribute, as listed in Table III. The syntax employed here is based on the notation for facts in the expert system scripting language CLIPS (JESS) which is also employed in the MFM Workbench. ms. For data exchange purposes, a simple graphoriented (entity-relationship) notation is available which internally is translated to the complex class. The simpler notation is also generated here and consists of generic typed nodes and three relations: link, whole-part and attribute, as listed in Table III. The syntax employed here is based on the notation for facts in the expert system scripting language CLIPS (JESS) which is also employed in the MFM Workbench.

TABLE III. FACT TYPES GENERATED TO REPRESENT MFM MODEL

\begin{tabular}{|c|c|}
\hline Fact type & Syntax \\
\hline model & (model file) \\
\hline node & (node function-type function-name) \\
\hline & (node link-type link-name) \\
\hline whole-part & (whole-part whole part) \\
\hline link & (link link-name begin function-name port-number) \\
\hline & (link link-name end function-name port-number ) \\
\hline attribute & (attribute attribute-type function-name parameter) \\
\hline & (attribute attribute-type function-name) \\
\hline & (attribute attribute-type function-namel function-name 2 ) \\
\hline
\end{tabular}

\section{Model Translation Algorithm}

The actual model translation is now a straightforward implementation of the patterns specified above. The function extract_data(), outlined in Algorithm 1, was implemented in the MATLAB scripting environment. In order to generate the MFM model Fact Base from the data given from the IEEE CDF model, the function analyzes the set of bus and branch data and generates a text-file with the corresponding facts for each element of the power system.

Four power system elements can be identified from the given data (generators, loads, transmission lines and transformer). Transmission lines and transformers are represented as seen in Figure 8b. The generators and loads are represented as noted in Section III.

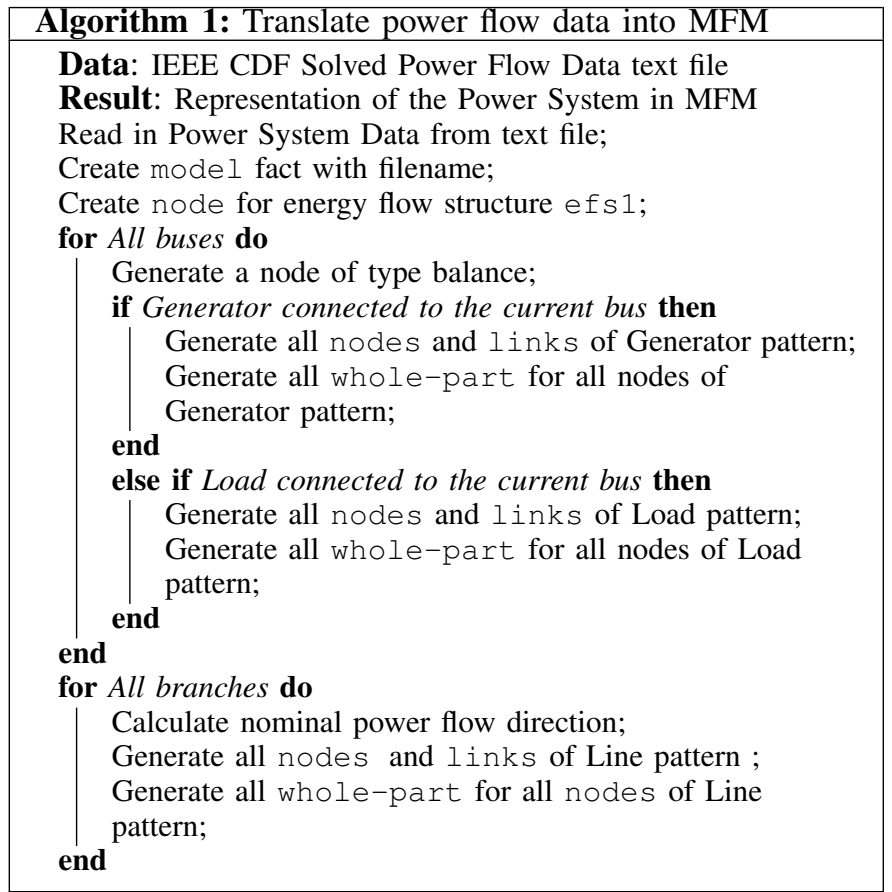

\section{CASE STUdies}

Two cases are considered for demonstration: $A$. the threebus case of Section III-A, Figure 3, and B. to an IEEE 300 Bus test system. The model files are generated and tested on a root-cause reasoning scenario using the MFM Workbench.

\section{A. Three-bus case}

The the translation algorithm was tested on the three-bus case introduced in Section III, which generated a model file of 136 lines and a structure as excerpted here:

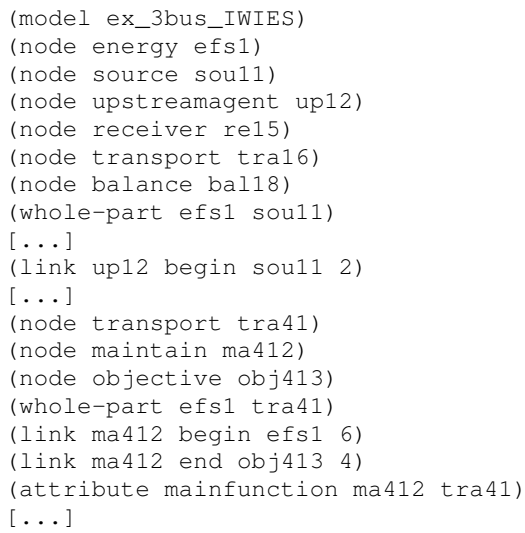

The resulting file was then opened inside MFM Workbench, which internally re-builds the model. To run a root-cause study (based on [13]), a disturbance scenario was specified with three observations of function states as input evidence. The root-cause algorithm computes five potential explanations ('causal paths') for sin31 not being supplied with energy. The evidence input and results are illustrated in Figure 10. The generated explanations can be relevant for diagnostic purposes, with several potential applications in the power systems domain. 


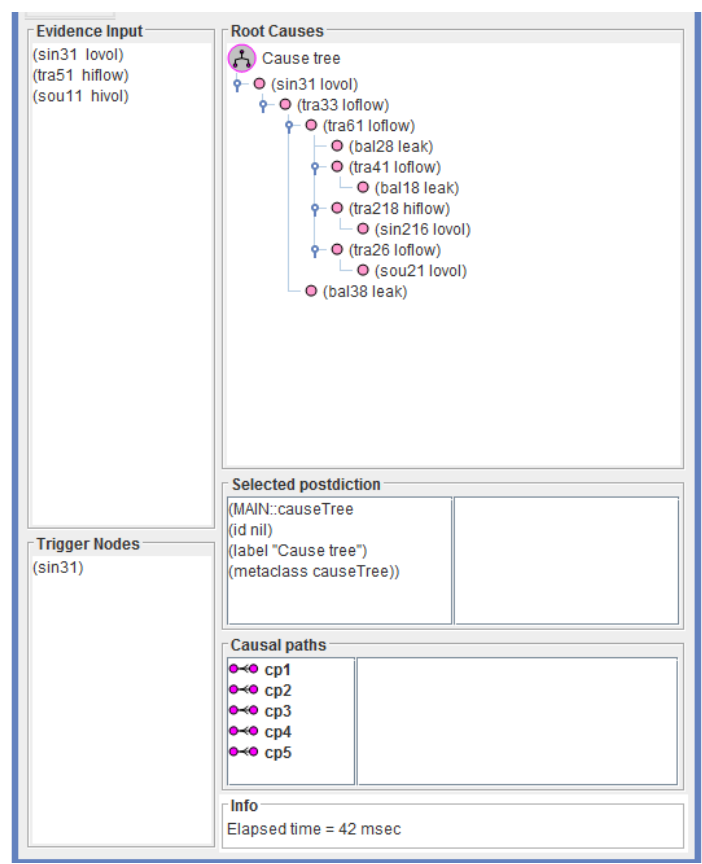

Fig. 10. Snapshot of the reasoning result for three-bus test case.

\section{B. 300 bus case}

The second case is a 300 bus test system (retrieved from http://www.ee.washington.edu/research/pstca/). This case demonstrates: $a$ ) the feasibility of a humanly intractable model size (14862 lines, 4118 nodes), as well as $b$ ) the fast reasoning which can be achieved with this specialized model structure (e.g. for 1 evidence [high flow in line from bus 77 to bus 80]: 33 causal paths, $139 \mathrm{~ms}$ ).

\section{CONCLUSION}

In this paper we have demonstrated how function-oriented system models suited for decision support can be generated directly from structured power system data. It has been shown how the available information contained in well-structured data can be enriched with further domain knowledge in corresponding MFM patterns and thus enhanced with decision relevant information. Using the simple IEEE common data format, it has been demonstrated that function-oriented system models can be generated directly from structured power system data, avoiding tedious and repetitive manual modeling and thereby enabling powerful new functions. The functionality of the resulting model and corresponding reasoning applications were illustrated on two case studies.

Some incremental challenges should be addressed in future work: a) the unique feature of MFM models resides with the ability to represent several layers of abstraction of the same physical structure - multiple coupled layers have not yet been generated; $b$ ) in the present implementation, the MFM patterns are hard-coded rather than linked to concepts of an ontology; an algorithm which only requires the high-level translation patterns as input would be desirable; $c$ ) different reasoning applications would be of interest, both to identify further relevant domain patterns and to establish the practical utility of MFMbased reasoning in power system applications, e.g. in reliability analysis, alarm filtering, decentralized decision-making; and $d$ ) the foundations of mapping data-sources to function-states and the nominal value identification, relations (2) to (5) in Figure 1 , can be supported by further methodological development.

The results presented here illustrate the foundations for a further application of semantic technologies toward engineering- and decision-support tools. The concept of application- and problem-oriented functional ontologies points into a direction with rich opportunities in application to intelligent energy systems.

\section{REFERENCES}

[1] "IEC 61850-ser: Communication networks and systems in substations - all parts." International Electrotechnical Commission, Geneva, IEC Standard, 2012, avalable at http://www.iec.ch.

[2] "IEC 61970-301: Energy management system application program interface (ems-api) - part 301: Common information model (cim) base," IEC Standard, 2003.

[3] S. Neumann, J. Britton, A. DeVos, and S. Widergren, "Use of the CIM ontology," Pacific Northwest National Laboratory, 2006.

[4] S. Rohjans et al., "Survey of smart grid standardization studies and recommendations," in First IEEE International Conference on Smart Grid Communications, 2010.

[5] T. Strasser, F. Andren, V. Vyatkin, G. Zhabelova, and C.-W. Yang, "Towards an IEC 61499 compliance profile for smart grids review and analysis of possibilities," in IECON 2012 - 38th Annual Conference on IEEE Industrial Electronics Society, 2012, pp. 3750-3757.

[6] F. Andren, M. Stifter, and T. Strasser, "Towards a semantic driven framework for smart grid applications: Model-driven development using CIM, IEC 61850 and IEC 61499," Informatik-Spektrum, vol. 36, no. 1, pp. 58-68, 2013. [Online]. Available: http: //dx.doi.org/10.1007/s00287-012-0663-y

[7] A. Saleem, Y. Wu, and L. Nordstrom, "Agent based real-time decision making in active distribution networks using iec61850 based functional ontology," in Proceedings of ISAP 2013, Tokyo, 2013, forthcoming.

[8] D. Marca and C. McGowan, SADT: structured analysis and design technique. McGraw-Hill Book Co., Inc.: New York, NY, 1988.

[9] M. Lind, "Plant modeling for human supervisory control," Transactions of the Institute of Measurement and Control, vol. 21, no. 4-5, pp. 171180, 1999.

[10] K. Heussen, "Control architecture modeling for future power systems," Ph.D. dissertation, Technical University of Denmark, 2011.

[11] _ - "Understanding control function and failure from a process perspective," in 2012 IEEE Workshop on Complexity in Engineering (COMPENG 2012), Aachen, Germany, Jun. 2012.

[12] K. Heussen and M. Lind, "On support functions for the development of MFM models," in Proceedings of STSS 2012, Okayama, 2012.

[13] M. Lind, "Reasoning about Causes and Consequences in Multilevel Flow Models," in ESREL 2011, Troyes, France, 2011.

[14] "61968-11 ed1.0: Application integration at electric utilities - system interfaces for distribution management - part 11: Common information model (cim) extensions for distribution," IEC, Tech. Rep., 2010.

[15] Working Group on a Common Format for Exchange of Solved Load Flow Data, "Common format for exchange of solved load flow data," IEEE Transactions on Power Apparatua and Systems, vol. PAS-92, no. 6, pp. 1916 - 1925, November 1973.

[16] M. Lind, "The use of flow models for automated plant diagnosis," in Human Detection and Diagnosis of System Failures, J. Rasmussen and W. B. Rouse, Eds. Plenum Press, New York, 1981.

[17] _ , "An introduction to multilevel flow modelling," International Journal of Nuclear Safety and Simulation, vol. 2, no. 1, 2011.

[18] - "Control Functions in MFM: Basic principles," International Journal of Nuclear Safety and Simulation, vol. 2, no. 2, 2011.

[19] K. Heussen, A. Saleem, and M. Lind, "Control architecture of power systems: Modeling of purpose and function," in Proceedings of the IEEE PES General Meeting 2009, 2009. 